\title{
Tanytarsini (Diptera: Chironomidae) of the Kashubian Lakeland
}

\author{
Wojciech GIŁKA and Patrycja DOMINIAK \\ Department of Invertebrate Zoology, University of Gdańsk, 81-378 Gdynia, Poland; \\ e-mail: scorpio@ocean.univ.gda.pl; e-mail:pdominitrox@interia.pl
}

\begin{abstract}
Chironomids of the tribe Tanytarsini of the Kashubian Lakeland (N Poland) are represented by 52 species, amounting over half of the Polish and almost third part of the European fauna. Most abundant species in the studied region are Cladotanytarsus mancus (Walker) and $C$. atridorsum Kieffer and the most frequent $-C$. mancus and Paratanytarsus inopertus (Walker). Adult Tanytarsini fly from the beginning of April to the second decade of October, reaching four peaks with a higher number of species during a season - spring, late spring, summer and late summer, with a maximum at the end of July. Most of species are recognized as poly- or bivoltine and three species as univoltine. Cladotanytarsus teres Hirvenoja, Micropsectra logani (Johannsen), Parapsectra styriaca (Reiss), Stempellinella flavidula (Edwards), Tanytarsus aberrans Lindeberg, T. multipunctatus Brundin and Thienemanniola ploenensis Kieffer, recorded in the Lakeland, are annotated as rare in Poland and Central Europe. Tanytarsus mancospinosus Ekrem \& Reiss and T. nigricollis Goetghebuer are new in the Polish fauna.
\end{abstract}

Key words: Diptera, Chironomidae, Tanytarsini, Kashubian Lakeland, Poland, faunistics, new records

\section{INTRODUCTION}

The Tanytarsini is a large, but still poorly known dipteran tribe of the family Chironomidae, grouping mainly those species that dwell and grow in freshwater habitats. The only comprehensive study based on data collected throughout Poland is a faunistic review of Polish Tanytarsini, the survey comprising information on geographic distribution and seasonal dynamics of the tribe (Giłka 2002). The data obtained hitherto from the Kashubian Lakeland show the region to support an exceptional Tanytarsini fauna, including northern and mountain species which are rarely found in Poland and Europe (Giłka 1997, 2001).

The present study focuses on the Tanytarsini of the Kashubian Lakeland, with a particular reference to the Kashubian Landscape Park and the Raduńskie Lakes, a group of the largest freshwater reservoirs in the area. The research presented was aimed at exploring the composition of the local Tanytarsini fauna and to provide additional data on biology of the rare species and those that have not been known from Poland to date.

\section{STUDY AREA}

The Kashubian Lakeland is the highest-lying (mean altitude $200 \mathrm{~m}$ a.s.l.) part of the Southern Baltic Lakelands sub-province. The Lakeland borders the Słupsk Plateau and Kashubian Coast to the north and east, while the Bytów Lakeland, Tuchola Forests, Starogard Lakeland, and Vistula Żuławy flanking the area to the west and south (Kondracki 1978, Augustowski 1979). This is the coldest mesoregion of the East-Pomeranian Lake District: the mean annual air temperature is about $7^{\circ} \mathrm{C}$ (about 17 and $-3^{\circ} \mathrm{C}$ being the mean monthly temperatures of July and January, respectively) (op. cit.). A considerable part $\left(3320 \mathrm{~km}^{2}\right)$ of 
the Kashubian Lakeland is occupied by the Kashubian Landscape Park with as much as $10.3 \%$ of the area of which being covered by water bodies (Przewoźniak 2000). The diverse relief, extensive altitude differences, and a complex system of lake troughs trending NNE and NE are a net result of Pleistocene glaciations, particularly the last one of them, the Baltic glaciation. The Park hosts as many as 48 water bodies larger than 1 ha; the largest of them are the flowthrough lakes of the upstream Radunia catchment: Lake Raduńskie Dolne and Lake Raduńskie Górne, which together with Lake Stężyckie, make up a 22-km long Radunia trough. The Radunia Lakes, situated at the altitude of about $162 \mathrm{~m}$ a.s.l., with a total shoreline length exceeding $48 \mathrm{~km}$, cover 1124.4 ha. The mean and maximum depths of Lake Raduńskie Dolne are 11.2 and $34.5 \mathrm{~m}$, respectively; the respective depths of Lake Raduńskie Górne are 15.5 and $43.0 \mathrm{~m}$. The lakes are mesotrophic, di- and tachymictic, their water being assigned to quality class II (Okulanis 1966, 1981; Jańczak 1997; Borowiak et al. 2000).

\section{MATERIAL AND METHODS}

The materials studied consisted of more than 6600 adult males of the Tanytarsini chironomids, which were identified. The specimens were collected in 1975-2006. Systematic collections were in 2001 and 2002 conducted at six sites on the Raduńskie Lakes. The adult Tanytarsini were being caught there from early April until the second decade of October, once a month in 2001 and twice a month in 2002. The insects were collected with a net and were attracted by light at 27 sites distributed within the Kashubian Lakeland, including 13 sites within the Kashubian Landscape Park and 8 on the Raduńskie Lakes. The sites were coded by the UTM grid quadrants (reported in parentheses): Babi Dół near Żukowo, at River Radunia (CF22); Borowo near Żukowo, at Lake Karlikowo (CF22); Borucino at Lake Raduńskie Górne (XA91); Borzestowo near Chmielno, at Lake Długie (XA92); Borzestowska Huta at Lake Raduńskie Dolne (CF02); Chrustowo at Lake Raduńskie Górne (XA91); Czarlino near Stężyca (XA81); Czysta Woda near Stężyca (XA81); Gołubie near Stężyca, at Lake Dąbrowskie (CF01); Gostomie near Stężyca (XA80); Kożyczkowo near Chmielno, at Lake Osuszyno (CF02); Lipowiec at Lake Raduńskie Dolne (CF02); Łączyno at Lake Raduńskie Dolne (CF01); Łubiana near Kościerzyna, at Lake Graniczne (XV89); Mirachowo near Chmielno on Mirachowska Stream (CF03); Niesiołowice near Stężyca, at lakes Długie and Skrzynka (XA81); Ogonki near Sulęczyno, at Lake Sumino (XA80); Osowa near Gdańsk, at Lake Wysockie (CF33); Otalżyno lake near Szemud (CF13); Pałubice near Sierakowice (XA83); Parchowski Młyn near Sulęczyno, at Lake Mausz (XA71); Przewóz at Lake Raduńskie Dolne (CF01); Stężyca at Lake Raduńskie Górne (XA91); Sulęczyno near Bytów, at Lake Guścierz Mały (XA81); Śnice near Stężyca, gravel-pit (XA90); Zgorzałe at Lake Raduńskie Górne (XA91); Żakowo near Sulęczyno, at Lake Martwe (XA81). Information on geographic distribution completed with recent records (Giłka 2006, Stur \& Ekrem 2006) follow the Fauna Europaea database (Sæther \& Spies 2004). The material examined is available in the Department of Invertebrate Zoology of University of Gdańsk.

\section{RESULTS}

The results, presented below, evidence the area studied to feature the highest Tanytarsini diversity in Poland. Of the 169 species recorded in Europe and 100 in Poland (Sæther \& Spies 2004; Giłka 2005, 2006; Giłka \& Abramczuk 2006; Stur \& Ekrem 2006), as many as 52 were found in the Kashubian Lakeland, including 42 in the Kashubian Landscape Park and 33 on the Raduńskie Lakes. The most abundant species in the Kashubian Lakeland included Cladotanytarsus mancus (Walker) (over 33\% examined specimens), C. atridorsum Kieffer (ca. 17\%), Micropsectra notescens (Walker) (ca. 6\%), Paratanytarsus inopertus (Walker) (ca. 6\%) and Tanytarsus sylvaticus 
(van der Wulp) (ca. $5 \%$ ), the first two being clearly dominant at the Raduńskie Lakes as well. The species most frequently recorded in the Lakeland included Cladotanytarsus mancus and Paratanytarsus inopertus, found at 13 out of the 27 sites sampled, as well as Micropsectra junci (Meigen) and Tanytarsus verralli Goetghebuer (found at 11 sites) and Paratanytarsus tenuis (Meigen) (10 sites). The species most frequently recorded on the Raduńskie Lake were Paratanytarsus inopertus and P. tenuis and also Tanytarsus usmaensis Pagast, T. mendax Kieffer and T. inaequalis Goetghebuer, found at 7 out of the 8 sites sampled (Table 1).

Table 1. Tanytarsini recorded in the Kashubian Lakeland, Kashubian Landscape Park and Lakes Raduńskie; $N-$ number of examined specimens, $k$ - number of sampling sites.

\begin{tabular}{|c|c|c|c|c|c|c|c|}
\hline \multirow[t]{2}{*}{ No. } & \multirow[t]{2}{*}{ Species } & \multicolumn{2}{|c|}{$\begin{array}{c}\text { Kashubian } \\
\text { Lakeland }\end{array}$} & \multicolumn{2}{|c|}{$\begin{array}{c}\text { Kashubian } \\
\text { Landscape Park }\end{array}$} & \multicolumn{2}{|c|}{ Lakes Raduńskie } \\
\hline & & $N$ & $k$ & $N$ & $k$ & $N$ & $k$ \\
\hline 1 & 2 & 3 & 4 & 5 & 6 & 7 & 8 \\
\hline 1 & Cladotanytarsus atridorsum Kieffer, 1924 & 1097 & 9 & 1073 & 7 & 1067 & 5 \\
\hline 2 & Cladotanytarsus difficilis Brundin, 1947 & 8 & 1 & - & - & - & - \\
\hline 3 & Cladotanytarsus mancus (Walker, 1856) & 2193 & 13 & 1581 & 8 & 1564 & 6 \\
\hline 4 & Cladotanytarsus nigrovittatus (Goetghebuer, 1922) & 14 & 3 & 2 & 1 & - & - \\
\hline 5 & Cladotanytarsus teres Hirvenoja, 1962 & 1 & 1 & 1 & 1 & - & - \\
\hline 6 & Cladotanytarsus wexionensis Brundin, 1947 & 9 & 4 & 4 & 2 & 4 & 2 \\
\hline 7 & Micropsectra apposita (Walker, 1856) & 124 & 5 & 124 & 5 & 25 & 4 \\
\hline 8 & Micropsectra atrofasciata (Kieffer, 1911) & 145 & 5 & 128 & 2 & 1 & 1 \\
\hline 9 & Micropsectra attenuata Reiss, 1969 & 25 & 2 & 3 & 1 & 3 & 1 \\
\hline 10 & Micropsectra junci (Meigen, 1818) & 95 & 11 & 43 & 8 & 31 & 5 \\
\hline 11 & Micropsectra logani (Johannsen, 1928) & 2 & 2 & 2 & 2 & 1 & 1 \\
\hline 12 & Micropsectra notescens (Walker, 1856) & 390 & 7 & 299 & 5 & 262 & 4 \\
\hline 13 & Micropsectra pallidula (Meigen, 1830) & 22 & 3 & 4 & 2 & - & - \\
\hline 14 & Micropsectra recurvata Goetghebuer, 1928 & 69 & 7 & 37 & 4 & 31 & 3 \\
\hline 15 & Parapsectra nana (Meigen, 1818) & 3 & 1 & 3 & 1 & - & - \\
\hline 16 & Parapsectra styriaca (Reiss, 1969) & 9 & 3 & 8 & 2 & 8 & 2 \\
\hline 17 & Paratanytarsus austriacus (Kieffer, 1924) & 1 & 1 & 1 & 1 & - & - \\
\hline 18 & Paratanytarsus bituberculatus (Edwards, 1929) & 117 & 8 & 82 & 4 & 79 & 2 \\
\hline 19 & Paratanytarsus dimorphis Reiss, 1965 & 147 & 7 & 108 & 6 & 92 & 5 \\
\hline 20 & Paratanytarsus dissimilis (Johannsen, 1905) & 68 & 6 & 68 & 6 & 52 & 5 \\
\hline 21 & Paratanytarsus inopertus (Walker, 1856) & 368 & 13 & 314 & 10 & 303 & 7 \\
\hline 22 & Paratanytarsus laetipes (Zetterstedt, 1850) & 22 & 4 & 7 & 3 & 7 & 3 \\
\hline 23 & Paratanytarsus lauterborni (Kieffer, 1909) & 7 & 4 & 7 & 4 & 7 & 4 \\
\hline 24 & Paratanytarsus tenellulus (Goetghebuer, 1921) & 4 & 2 & 4 & 2 & 3 & 1 \\
\hline 25 & Paratanytarsus tenuis (Meigen, 1830) & 134 & 10 & 114 & 7 & 114 & 7 \\
\hline 26 & Rheotanytarsus curtistylus (Goetghebuer, 1921) & 170 & 3 & 30 & 2 & 1 & 1 \\
\hline 27 & Rheotanytarsus pentapoda (Kieffer, 1909) & 2 & 1 & - & - & - & - \\
\hline 28 & Rheotanytarsus ringei Lehmann, 1970 & 142 & 1 & 142 & 1 & - & - \\
\hline 29 & Stempellina bausei (Kieffer, 1911) & 42 & 1 & 42 & 1 & 42 & 1 \\
\hline 30 & Stempellina subglabripennis (Brundin, 1947) & 6 & 1 & - & - & - & - \\
\hline 31 & Stempellinella edwardsi Spies \& Sæther, 2004 & 3 & 2 & 1 & 1 & - & - \\
\hline 32 & Stempellinella flavidula (Edwards, 1929) & 1 & 1 & - & - & - & - \\
\hline 33 & Tanytarsus aberrans Lindeberg, 1970 & 1 & 1 & - & - & - & - \\
\hline 34 & Tanytarsus debilis (Meigen, 1830) & 14 & 5 & 14 & 5 & 14 & 5 \\
\hline 35 & Tanytarsus dibranchius Kieffer, 1926 & 6 & 1 & - & - & - & - \\
\hline 36 & Tanytarsus excavatus Edwards, 1929 & 54 & 6 & 30 & 4 & 15 & 2 \\
\hline 37 & Tanytarsus gregarius Kieffer, 1909 & 1 & 1 & 1 & 1 & - & - \\
\hline 38 & Tanytarsus heusdensis Goetghebuer, 1923 & 1 & 1 & - & - & - & - \\
\hline 39 & Tanytarsus inaequalis Goetghebuer, 1921 & 120 & 8 & 119 & 7 & 119 & 7 \\
\hline 40 & Tanytarsus mancospinosus Ekrem \& Reiss, 1999 & 1 & 1 & 1 & 1 & 1 & 1 \\
\hline 41 & Tanytarsus medius Reiss et Fittkau, 1971 & 2 & 2 & 1 & 1 & 1 & 1 \\
\hline 42 & Tanytarsus mendax Kieffer, 1925 & 182 & 8 & 181 & 7 & 181 & 7 \\
\hline 43 & Tanytarsus miriforceps (Kieffer, 1921) & 14 & 3 & 14 & 3 & 14 & 3 \\
\hline 44 & Tanytarsus multipunctatus Brundin, 1947 & 10 & 1 & - & - & - & - \\
\hline
\end{tabular}




\begin{tabular}{|c|c|c|c|c|c|c|c|}
\hline 1 & 2 & 3 & 4 & 5 & 6 & 7 & 8 \\
\hline 45 & Tanytarsus nemorosus Edwards, 1929 & 1 & 1 & 1 & 1 & - & - \\
\hline 46 & Tanytarsus nigricollis Goetghebuer, 1939 & 1 & 1 & - & - & - & - \\
\hline 47 & Tanytarsus occultus Brundin, 1949 & 129 & 5 & 124 & 3 & 124 & 3 \\
\hline 48 & Tanytarsus pallidicornis (Walker, 1856) & 4 & 3 & 3 & 2 & 1 & 1 \\
\hline 49 & Tanytarsus sylvaticus (van der Wulp, 1858) & 333 & 5 & 201 & 4 & 201 & 4 \\
\hline 50 & Tanytarsus usmaensis Pagast, 1931 & 94 & 9 & 83 & 7 & 83 & 7 \\
\hline 51 & Tanytarsus verralli Goetghebuer, 1928 & 196 & 11 & 102 & 8 & 89 & 6 \\
\hline 52 & Thienemanniola ploenensis Kieffer, 1921 & 35 & 1 & - & - & - & - \\
\hline & Total & 6639 & 27 & 5107 & 13 & 4540 & 8 \\
\hline & Number of species & \multicolumn{2}{|c|}{52} & \multicolumn{2}{|c|}{42} & \multicolumn{2}{|c|}{33} \\
\hline
\end{tabular}

The seasonal distribution of adult Tanytarsini in the Kashubian Lakeland is well comparable with previous observations (Giłka 2001) and completes results obtained from whole Poland (Giłka 2002). A flight period of the adult Tanytarsini in the Kashubian Lakeland starts in the beginning of April and lasts to the second decade of October (Table 2). At least four peaks of abundance with a distinctly higher number of species were observed during a season. The spring peak occurs in April and includes early spring species of the genera Thienemanniola Kieffer, Micropsectra Kieffer and Tanytarsus van der Wulp. Most of species start their flight in May reaching a late spring peak in the beginning of June. The next generation (or generations) occurs in summer, reaching maximum at the end of July (summer peak). At least 18 species can occur at the first two decades of September (late summer peak), of which only a few are still recorded in October. Three species recorded in the Kashubian Lakeland were recognized as spring univoltine (Th. ploenensis Kieffer, Tanytarsus miriforceps Kieffer and T. sylvaticus (van der Wulp)). Basing on records from other sites in Poland and Central European countries, six species (Parapsectra nana Meigen, Paratanytarsus austriacus (Kieffer), P. lauterborni (Kieffer), Tanytarsus multipunctatus Brundin, T. nemorosus Edwards and $T$. nigricollis Goetghebuer) were recognized as producing probably more than one generation per year. Most of species known from the Kashubian Lakeland are bi- or polyvoltine ( 35 species), producing at least two, three or four generations per year (Table 2, Fig. 1).

\section{Annotations on rare and new species in Poland}

\section{Cladotanytarsus teres Hirvenoja, 1962}

Material examined: $1 \hat{\delta}$, Kożyczkowo near Chmielno, netting at Lake Osuszyno, 1 June 1997, leg. E. Sontag.

Distribution and biology: Data on this northern species have been provided by Giłka (1997). The Kashubian Lakeland is the only area inhabited by C. teres outside of the Scandinavian Peninsula.

\section{Micropsectra logani (Johannsen, 1928)}

Material examined: $1 \hat{\jmath}$, Lipowiec, netting, 12 May 2002, leg. P. Dominiak; $1 \hat{\sigma}$ Mirachowo, netting on helocrene,16 April 1998, leg. W. Giłka.

Distribution and biology: M. logani is a Holarctic species, known mostly from the lakes of Fennoscandia (Brundin 1949, as M. groenlandica Andersen; Stur \& Ekrem 2006) and seldom reported from central Europe, mostly from the profundal of deep lakes and from rivers. In the Kashubian Lakeland, the adults were caught at helocrenic habitats in April and May. In addition to the area sampled in this study, M. logani is known in other four sites in Poland: from the Kujawy (adults collected in July), the Mazurian Lake District, and from the Świętokrzyskie Mountains, where the larvae were found in a muddy sediment lining a lenitic section of a river (Giłka 2002, as M. groenlandica). In Poland, M. logani produces at least two generations per year. 
Table 2. Seasonal distribution of adult Tanytarsini in the Kashubian Lakeland: • - Lakes Raduńskie, • - remaining data from the Kashubian Lakeland.

\begin{tabular}{|c|c|c|c|c|c|c|c|c|c|c|c|c|c|c|c|c|c|c|c|c|c|c|}
\hline \multirow{2}{*}{ No. } & \multirow{2}{*}{ Species/period } & \multicolumn{3}{|c|}{ April } & \multicolumn{3}{|c|}{ May } & \multicolumn{3}{|c|}{ June } & \multicolumn{3}{|c|}{ July } & \multicolumn{3}{|c|}{ August } & \multicolumn{3}{|c|}{ Sept. } & \multicolumn{3}{|c|}{ Oct. } \\
\hline & & 1 & 2 & 3 & 1 & 2 & 3 & 1 & 2 & 3 & 1 & 2 & 3 & 1 & 2 & 3 & 1 & 2 & 3 & 1 & 2 & 3 \\
\hline 1 & C. atridorsum & & & & & $\bullet$ & $\bullet$ & $\bullet$ & 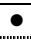 & $\bullet$ & $\circ$ & 10 & $\bullet$ & $\bullet$ & $1^{\circ}$ & $\bullet$ & $\bullet$ & & & & & \\
\hline 2 & C. difficilis & & & & & & & 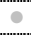 & & & & & & & & & & & & & & \\
\hline 3 & C. mancus & & & & 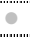 & $\bullet$ & $\bullet$ & $\bullet$ & $\bullet$ & & $\bullet$ & $\bullet$ & $\bullet$ & & & $\bullet$ & $\bullet$ & 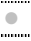 & $\bullet$ & & & \\
\hline 4 & C. nigrovittatus & & & & & & 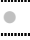 & 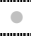 & & & . & & 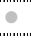 & & & & & & & & & \\
\hline 5 & C. teres & & & & & & & $\circ$ & & & & & & & & & & & & & & \\
\hline 6 & C. wexionensis & & & & & & $\bullet$ & $\bullet$ & & & (3) & 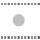 & & & & & & 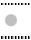 & & & & \\
\hline 7 & M. apposita & $\bullet$ & $\bullet$ & $\bullet$ & - & & & $\bullet$ & $\bullet$ & (n) & $\bullet$ & & $\bullet$ & $\bullet$ & 0 & & $\bullet$ & 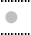 & $\bullet$ & & & \\
\hline 8 & M. atrofasciata & & $\bullet$ & & 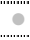 & & & 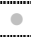 & 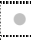 & & 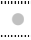 & & & & $\circ$ & & 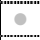 & 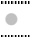 & 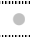 & & & \\
\hline 9 & M. attenuata & & & & & & & & 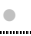 & & & & $\bullet$ & & & & $\bullet$ & $\circ$ & $\bullet$ & & & \\
\hline 10 & M. junci & $\bullet$ & $\bullet$ & $\bullet$ & 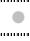 & & $\bullet$ & $\bullet$ & & & 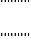 & & $\bullet$ & $\bullet$ & 0 & & $\bullet$ & $\circ$ & $\bullet$ & & & \\
\hline 11 & M. logani & & $\stackrel{\circ}{\circ}$ & & & $\bullet$ & & & & & & & & & & & & & & & & \\
\hline 12 & M. notescens & $\bullet$ & 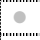 & $\bullet$ & 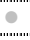 & $\bullet$ & & - & ............ & & $\bullet$ & & & & $\bullet$ & & $\bullet$ & $\circ$ & & & & \\
\hline 13 & M. pallidula & & (1) & & & & (3)!-" & & & & & & & & & & & 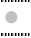 & & & & \\
\hline 14 & M. recurvata & $\bullet$ & $\bullet$ & $\bullet$ & 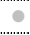 & & & & $\bullet$ & & $\bullet$ & 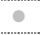 & $\bullet$ & & & & & 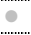 & 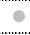 & & & \\
\hline 15 & P. nana & & & & & & 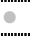 & & & & & & & & & & & & & & & \\
\hline 16 & P. styriaca & & & & & $\bullet$ & & $\circ$ & & & & & $\bullet$ & & & & & & & & & \\
\hline 17 & P. austriacus & & 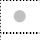 & & & & & & & & & & & & & & & & & & & \\
\hline 18 & P. bituberculatus & & & & 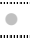 & & & & & & (3) & $+\infty$ & 10 & . & & .-" & $\bullet$ & (3)."... & $\bullet$ & 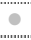 & & \\
\hline 19 & P. dimorphis & & & & & $\bullet$ & & & $\bullet$ & 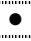 &..... & 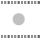 & $\bullet$ & $\bullet$ & 0 & $\bullet$ & & (3) & & (3) & & \\
\hline 20 & P. dissimilis & & & & 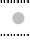 & $\bullet$ & $\bullet$ & & $\bullet$ & & . & 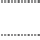 & $\bullet$ & $\bullet$ & $\bullet$ & & & 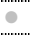 & $\bullet$ & & & \\
\hline 21 & P. inopertus & & & & 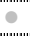 & $\bullet$ & $\bullet$ & $\bullet$ & $\bullet$ & $\bullet$ & $\bullet$ & $\bullet$ & $\bullet$ & $\bullet$ & $\bullet$ & $\circ$ & $\bullet$ & 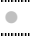 & $\bullet$ & 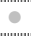 & $\bullet$ & \\
\hline 22 & P. laetipes & & & & $\ldots$ & & & & $\bullet$ & & s"men & 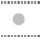 & $\bullet$ & $\bullet$ & $\bullet$ & $\bullet$ & $\bullet$ & & & s'm & & \\
\hline 23 & P. lauterborni & & & & 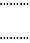 & & & & & & & & $\bullet$ & $\bullet$ & & & $\bullet$ & & 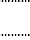 & (3) & & \\
\hline 24 & P. tenellulus & & & & 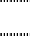 & $\bullet$ & & & & & 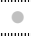 & & " & $\bullet$ & & & - & & & . & & \\
\hline 25 & P. tenuis & & & & (3) & $\bullet$ & $\bullet$ & & & & $\bullet$ & $\bullet$ & $\bullet$ & $\bullet$ & $\bullet$ & $\bullet$ & $\bullet$ & & $\bullet$ & (3) & & \\
\hline 26 & Rh. curtistylus & & & & 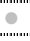 & 0 & & & & & & & $\bullet$ & & & & 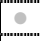 & $\circ$ & & & & \\
\hline 27 & Rh. pentapoda & & & & 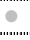 & & & & & & & & & & & & & & & & & \\
\hline 28 & $R h$. ringei & & & & (3) & & & & & & 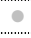 & & & & 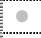 & & & $\circ$ & & & & \\
\hline 29 & S. bausei & & & & & $\bullet$ & & & & & . & & & & & & & & & & & \\
\hline 30 & S. subglabripennis & & & & 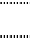 & & & & & & (3) & 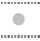 & & & & & & & & & & \\
\hline 31 & S. edwardsi & & & & 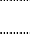 & & & 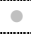 & & & 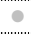 & & & & & & & & & & & \\
\hline 32 & S. flavidula & & & & (n) & & & & & & & & & & & & & & & & & \\
\hline 33 & T. aberrans & & & & 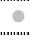 & & & & & & & & & & & & & & & & & \\
\hline 34 & T. debilis & & & & & & & $\bullet$ & & & $\bullet$ & $\bullet$ & $\bullet$ & $\bullet$ & & $\bullet$ & & & & & & \\
\hline 35 & T. dibranchius & & & & & & & & & & & & $\circ$ & & & & & & & & & \\
\hline 36 & T. excavatus & & & & & & $\bullet$ & 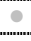 & $\bullet$ & 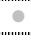 & 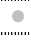 & $\bullet$ & $\bullet$ & 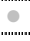 & $\bullet$ & & & & & & & \\
\hline 37 & T. gregarius & & & & & & 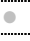 & & & & & & & & & & & & & & & \\
\hline 38 & T. heusdensis & & & & 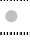 & & & & & & & & & & & & & & & & & \\
\hline 39 & T. inaequalis & & & & & & $\bullet$ & $\bullet$ & $\bullet$ & $\bullet$ & $\bullet$ & $\bullet$ & $\bullet$ & $\bullet$ & & & $\bullet$ & & $\bullet$ & (3) & & \\
\hline 40 & T. mancospinosus & & & & & & & & & & & & & & & & & & & & $\bullet$ & \\
\hline 41 & T. medius & & & & & & & & & & & & $\bullet$ & & & & & & & & & \\
\hline 42 & T. mendax & & & & & $\bullet$ & $\bullet$ & $\bullet$ & $\bullet$ & $\bullet$ & $\bullet$ & $\bullet$ & $\bullet$ & $\bullet$ & $\bullet$ & $\bullet$ & $\bullet$ & & $\bullet$ & & $\bullet$ & \\
\hline 43 & T. miriforceps & & $\bullet$ & $\bullet$ & & & & & & & & & & & & & & & & & & \\
\hline 44 & T. multipunctatus & & & & & & & & & & 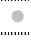 & & & & 0 & $\circ$ & & & & & & \\
\hline 45 & T. nemorosus & & & & & & - & 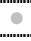 & & & 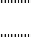 & & & & 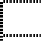 & & & & & & & \\
\hline 46 & T. nigricollis & & & & & $\circ$ & & & & & & & & & & & & & & & & \\
\hline 47 & T. occultus & & & & & & & $\bullet$ & $\bullet$ & & & 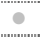 & $\bullet$ & $\bullet$ & $\bullet$ & $\bullet$ & $\bullet$ & & $\bullet$ & & $\bullet$ & \\
\hline 48 & T. pallidicornis & & & $\bullet$ & & & & & ......... & & & & & & 0 & & & 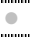 & & & & \\
\hline 49 & T. sylvaticus & ㅇ...... & 0 & $\bullet$ & & $\bullet$ & & & & & & & & & & & & & & & & \\
\hline 50 & T. usmaensis & & & & & $\bullet$ & $\bullet$ & $\bullet$ & $\bullet$ & $\bullet$ & $\bullet$ & $\bullet$ & $\bullet$ & $\bullet$ & $\bullet$ & & $\bullet$ & $\circ$ & $\bullet$ & 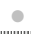 & $\bullet$ & \\
\hline 51 & T. verralli & & & & & & $\bullet$ & $\bullet$ & ........ & $\bullet$ & $\bullet$ & $\bullet$ & $\bullet$ & $\bullet$ & $\bullet$ & $\bullet$ & $\bullet$ & $\circ$ & & & & \\
\hline 52 & Th. ploenensis & & $\circ$ & & & & & & & & & & & & & & & & & & & \\
\hline
\end{tabular}




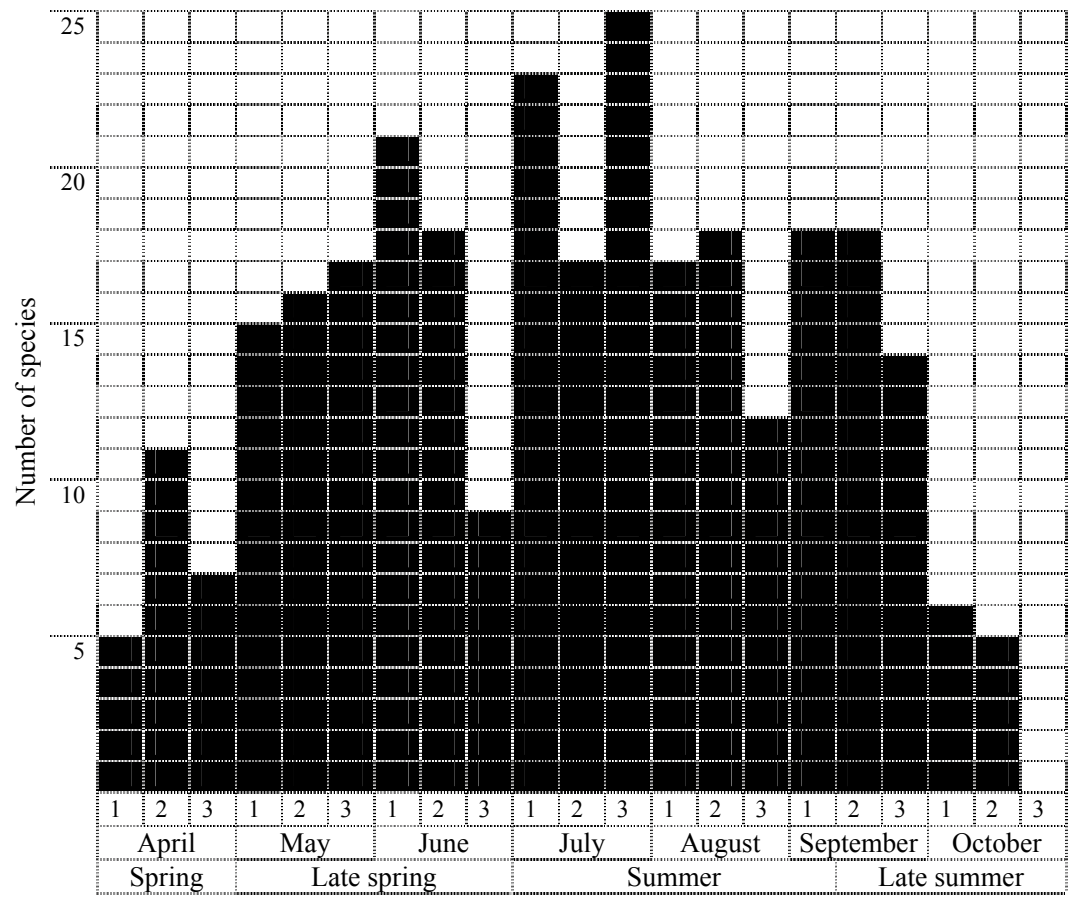

Fig. 1. Diagram of seasonal distribution of adult Tanytarsini in the Kashubian Lakeland

\section{Parapsectra styriaca (Reiss, 1969)}

Material examined: $2 \widehat{\jmath}$, Borzestowska Huta, netting, 30 July 2001, leg. P. Dominiak;

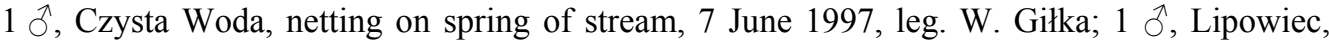
netting, 12 May 2001, leg. P. Dominiak; $4 \hat{\jmath} \widehat{\jmath}$, netting, 30 July 2001, leg. P. Dominiak; $1 \hat{\jmath}$, netting, 12 May 2002, P. Dominiak. Additional data: 1 đे, Czarna Hańcza near Słupie, netting, at peat bog, 24 May 2006, leg. P. Dominiak.

Distribution and biology: P. styriaca is a rarely reported species, known primarily from mountains and uplands in western and central Europe. Its oligo- and stenothermal larvae develop in lakes, swamps and springs (Reiss 1969, Langton 1991). In Poland, P. styriaca emerges at least twice per season. The species was collected from May until the end of July, mainly at the helocrene and peat bogs. In addition to the Kashubian Lakeland, the Polish records of $P$. styriaca originate from the region of Suwałki (NE Poland).

\section{Stempellinella flavidula (Edwards, 1929)}

Material examined: 1 đ, Babi Dól, reserve "Jar Rzeki Raduni”, netting on river, 8 May 1983, leg. R. Szadziewski.

Distribution and biology: Solitary records of $S$. flavidula originate from sites scattered throughout Europe. The habitats supporting larval developments include lakes and small stagnant water bodies as well as trickles, rivulets and springs (Langton 1991). The only specimen of $S$. flavidula known from Poland was found in sample containing abundant males of Rheotanytarsus curtistylus (Goetghebuer), collected from a gorge-forming section of River Radunia. 
Tanytarsus aberrans Lindeberg, 1970

Material examined: 1 ô, Borowo, netting, 10 May 1997, leg. E. Kaczorowska.

Distribution and biology: T. aberrans is a limnophilous species known primarily from oligotrophic lakes of the Scandinavian Peninsula. The species has also been collected at sites scattered in central Europe, mainly in uplands and mountains. Adults and pupal exuviae of $T$. aberrans were recorded from May until August (Koskenniemi \& Paasivirta 1987, Bitušík 1996). The male specimen found in this study was caught in May at a small eutrophic lake. It may be presumed that, in Poland, the species appears as imago also later in the season.

\section{Tanytarsus mancospinosus Ekrem \& Reiss, 1999}

Material examined: $1 \overbrace{}^{\lambda}$, Chrustowo, netting, 20 Oct. 2001, P. Dominiak. Additional data: $2 \widehat{\jmath} \hat{\jmath}$, Southern Baltic Coastlands, Choczewskie Lake near Żarnowiec (XA86), netting, 18 June 1979, leg. R. Szadziewski.

Distribution and biology: T. mancospinosus is a recently discovered species that develops in meso- and eutrophic lakes. In Germany and Denmark, adults of this species emerge from June until August (Ekrem et al. 1999). In Poland, T. mancospinosus was recorded at a shallow embayment of the Raduńskie Lake in the second decade of October. Outside of the Kashubian Lakeland it was found in mid-June. This is the first Polish record of T. mancospinosus.

\section{Tanytarsus multipunctatus Brundin, 1947}

Material examined: Żakowo, netting at Lake Martwe, leg. E. Kaczorowska: 4 ๙ึે, 22

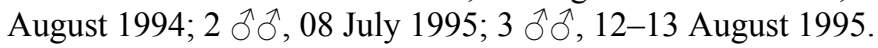

Distribution and biology: The species is known from oligotrophic lakes of central Europe and Fennoscandia where it was reported in May as well as from July until September (Brundin 1947, Ekrem 2004). The males examined in this study were caught in the closest proximity to a small eutrophic lake in July and August. It may be expected that the species occurs in Poland also in spring period. The Kashubian Lakeland is the only area of T. multipunctatus occurrence known so far in Poland.

\section{Tanytarsus nigricollis Goetghebuer, 1939}

Material examined: $1 \delta$, Śnice, netting at gravel-pit, 13 May 2006, leg. W. Giłka \& J. Naumowicz.

Distribution and biology: T. nigricollis is a rare and poorly known species reported from a few sites in western Europe. Its larvae inhabit lakes (Goetghebuer 1939, Reiss \& Fittkau 1971). In this study, the species was collected from the vicinity of submerged gravel-pit together with an abundant sample consisting of Tanytarsus excavatus Edwards. The German and Belgian data indicate $T$. nigricollis to produce at least two generations per year, as the species was recorded in mid-May and mid-June as well as in late September (op. cit.). This is the first record of T. nigricollis in Poland.

\section{Thienemanniola ploenensis Kieffer, 1921}

Material examined: 20 $\widehat{\partial}$ and 2 q $ᄋ$ adults, pairs in copula, pupae, Niesiołowice, at bank, water surface, 17 April 1998; $11 \hat{\jmath}, 2$ $ᄋ+$, water surface, plants and stones at bank, 18 April

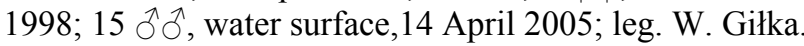

Distribution and biology: Th. ploenensis is a European temperate species, developing in fresh waters, from lakes to small natural and artificial water bodies (Lehmann 1973, Janecek 1995). Th. ploenensis produces a single, spring generation of adults. They lack the ability to fly and glide on the water surface by using their shortened wings and strongly elongated fore legs set wide apart. Wave action and air jets enhance concentrations of adults and push them towards the shore. Some specimens were observed transported by the wind above the water surface. The Th. ploenensis adults aggregate on riparian plants and boulders in search for 
a mating partner. Copulating pairs were observed, as well copulation attempts of male-male pairs. A small eutrophic lake in Niesiołowice is one of the few known European sites of $T h$. ploenensis.

\section{ACKNOWLEDGEMENTS}

We thank collectors: Ryszard Szadziewski, Elżbieta Kaczorowska, Elżbieta Sontag and Justyna Naumowicz (University of Gdańsk) for making the material available to us; anonymous reviewers for comments on earlier version of the manuscript, and Jolanta Wytwer (Museum and Institute of Zoology, Polish Academy of Sciences, Warszawa) for improvements of our paper.

\section{REFERENCES}

Augustowski B. 1979. Pojezierze Kaszubskie. Ossolineum, Gdańsk, 602 pp.

BITUŠ́́K P. 1996. Some chironomids (Diptera, Chironomidae) new to Slovakia. Biologia, Bratislava, 51: 583-584.

BOROWIAK D., LANGE W. \& MAŚlANKA W. 2000. Przyrodnicze uwarunkowania ochrony cieków i jezior. In: PRZEWOŹNIAK M. (ed.), Materiały do monografii przyrodniczej regionu gdańskiego. Tom 2. Kaszubski Park Krajobrazowy: walory, ochrona, zagrożenia, pp. 45-74. Marpress, Gdańsk, 256 pp.

BRUNDIN L. 1947. Zur Kenntnis der Swedischen Chironomiden. Arkiv för Zoologi 39: 1-95.

BRUNDIN L. 1949. Chironomiden und andere Bodentiere südschwedischen Urgebirgseen. Ein Bietrag zur Kenntnis der boden-faunistischen Charakterzüge schwedischen oligotropher Seen. Reports of the Institute for Freshwater Research, Drottningholm, 30: 1-915.

EKREM T. 2004. Immature stages of European Tanytarsus species I. The eminulus-, gregarius-, lugens- and mendax species groups (Diptera, Chironomidae). Mitteilungen aus dem Museum für Naturkunde in Berlin, Deutsche Entomologische Zeitschrift 51: 97-146.

EKREM T., REISS F. \& LANGTON P. H. 1999. Tanytarsus mancospinosus sp.n. (Diptera: Chironomidae) from eutrophic lakes in Europe. Norwegian Journal of Entomology 46: 79-87.

GiŁKA W. 1997. Cladotanytarsus teres in Poland (Diptera: Chironomidae). Polskie Pismo Entomologiczne 66: 271-276.

GıŁKA W. 2001. Seasonal dynamic of some chironomids of the tribe Tanytarsini in Kashubian Lakeland (Diptera: Chironomidae). Acta Entomologica Silesiana 7/8: 31-42.

GıŁKA W. 2002. Tanytarsini (Diptera: Chironomidae) of Poland - a faunistic review. Polskie Pismo Entomologiczne 66: $271-276$.

GıŁKA W. 2005. A systematic review of European Stempellina Thienemann et Bause (Diptera: Chironomidae) with description of a new species from Fennoscandia. Annales Zoologici 55: 413-419.

GıŁKA W. 2006. Setny gatunek z plemienia Tanytarsini w polskiej faunie (Diptera: Chironomidae). Dipteron. Bulletin of the Dipterological Section of the Polish Entomological Society 22: 8-10.

GilKA W. \& ABRAMCZUK Ł. 2006. Micropsectra davigra sp. n. from the Tatra Mountains - a contribution to the systematics of the Micropsectra attenuata species group (Diptera: Chironomidae). Polskie Pismo Entomologiczne 75: $39-44$

Goetghebuer M. 1939. Ceratopogonidae et Chironomidae nouveaux ou peu connus d'Europe (Nouvième note). Bulletin et Annales de la Société Royale d'Entomologique de Belgique 79: 219-229.

JANECEK B. F. U. 1995. Tanytarsus niger ANDERSEN (Diptera: Chironomidae) and the chironomid community in Gebhartsteich, a carp pond in northern Austria. In: CRANSTON P.S. (ed.), Chironomids: from genes to ecosystems, pp. 281-296. Proc. $12^{\text {th }}$ Int. Symp. on Chironomidae, Canberra, January $23-26$, 1994. CSIRO Australia, East Melbourne, $482 \mathrm{pp}$.

JAŃCZAK J. 1997. Atlas Jezior Polski. Jeziora zlewni rzek Przymorza i dorzecza dolnej Wisły. Vol. 2. Bogucki, Poznań, $256 \mathrm{pp}$

KoskenNiemi E. \& PAAsivirTa L. 1987. The chironomid (Diptera) fauna in a Finnish reservoir during its first four years. Entomologica scandinavica, Suppl. 29: 239-246.

KONDRACKi J. 1978. Geografia fizyczna Polski. PWN, Warszawa, 435 pp.

LANGTON P.H. 1991. A key to pupal exuviae of West Palaearctic Chironomidae. P.H. Privately published by P.H. Langton, Graytones, Peterborough, 386 pp.

LEHMANN J. 1973. Systematik und phylogenetische Studie über die Gattung Thienemanniola KIEFFER und Corynocera ZETTERSTEDT (Diptera: Chironomidae). Hydrobiolgia 43: 381-414.

OKULANIS E. 1966. Morfologia i batymetria Jezior Raduńskich. Zeszyty Geograficzne WSP w Gdańsku 8: 61-287.

OKULANIS E. 1981. Studium limnologiczne Jezior Raduńsko-Ostrzyckich. Ossolineum, Gdańsk, 108 pp. 
PRZEWOŹNIAK M. 2000. Diagnoza stanu Kaszubskiego Parku Krajobrazowego - problemy trójochrony (przyrodakultura-krajobrazy). In: PRZEWOŹNIAK M. (ed.), Materiały do monografii przyrodniczej regionu gdańskiego. 2. Kaszubski Park Krajobrazowy: walory, ochrona, zagrożenia, pp. 9-22. Marpress, Gdańsk, 256 pp.

ReISs F. 1969. Revision der Gattung Micropsectra KIEFF., 1909 (Diptera, Chironomidae). 1 - Die attenuata - Gruppe der Gattung Micropsectra. Beschreibung 5 neuer arten aus Mitteleuropa und Nordafrika. Deutsche Entomologische Zeitschrift 16: 431-449.

REISS F. \& FITTKAU E. J. 1971. Taxonomie und Ökologie europäisch verbreiteter Tanytarsus-Arten (Chironomidae, Diptera). Archiv für Hydrobiologie, Suppl. 40: 75-200.

SÆTher O. A. \& SPIES M. 2004. Chironomidae. In: Fauna Europaea Service, Fauna Europaea version 1.1. Internet database available online: http://www.faunaeur.org (date of access: 10th May 2007).

STUR E. \& EKREM T. 2006. A revision of West Palaearctic species of the Micropsectra atrofasciata species group (Diptera: Chironomidae). Zoological Journal of the Linnean Society 146: 165-225.

\section{STRESZCZENIE}

\section{[Tanytarsini (Diptera: Chironomidae) Pojezierza Kaszubskiego]}

Na Pojezierzu Kaszubskim odnotowano 52 gatunki Tanytarsini, z których 42 wykazano na obszarze Kaszubskiego Parku Krajobrazowego, a 33 nad Jeziorami Raduńskimi. Charakterystyczne cechy klimatu oraz ukształtowanie terenu na Pojezierzu Kaszubskim sprzyjają występowaniu zimnolubnych gatunków północnych i górskich (Cladotanytarsus teres, Micropsectra logani, Parapsectra styriaca). Tanytarsus mancospinosus i Tanytarsus nigricollis to gatunki dotychczas nie notowane w Polsce. Cladotanytarsus teres, Micropsectra logani, Parapsectra styriaca, Stempellinella flavidula, Tanytarsus aberrans, T. multipunctatus i Thienemanniola ploenensis to gatunki rzadkie, znane w środkowej Europie z nielicznych stanowisk. Na Pojezierzu Kaszubskim postaci dorosłe Tanytarsini pojawiają się od początku kwietnia do drugiej dekady października, przy wyraźnie wyższej liczbie gatunków w połowie kwietnia, na początku czerwca, w końcu lipca (maksimum) i w pierwszej połowie września. Większość spośród notowanych gatunków należy do wielopokoleniowych, wydających dwa lub więcej pokoleń w sezonie. Imagines trzech gatunków pojawiały się tylko wiosną Thienemanniola ploenensis, Tanytarsus miriforceps i T. sylvaticus. 